\title{
Partitioning of digital images using inclusion hierarchies with contraction mapping
}

\author{
Neelam Yadav \\ Research Scholar, Amity School of Applied Sciences, Amity University \\ Lucknow, India \\ neelam3376@yahoo.co.in \\ Piyush Kumar Tripathi \\ Associate Professor, Amity School of Applied Sciences, Amity University \\ Lucknow, India \\ pktripathi@lko.amity.edu \\ Sajjan Lal Maurya \\ Assistant Professor, Department of Applied Sciences, F.G.I.E.T., \\ Raebareli, India \\ slmauryafgiet@rediffmail.co
}

\begin{abstract}
High-quality images often involve large pixel data and more space for storage and thus not transmittable rapidly. Such an image data inter alia compressed video files and streams constitute significant share in internet traffic. Various image compression techniques have been developed primarily using contraction mapping that subject image to partitioning in domain and range blocks. Specialized fractal image compression methods then exploit the self similarity property of the image as a whole or its parts. Thus, the partitioning strategy finds a crucial role in achieving better rate of data compression with acceptable quality of the output image. This paper highlights few partitioning methods and subsequently defines the partitioning curve with example from utility point. Further, a novel and efficient partitioning method is proposed taking into account the centre of distribution of the clusters with contraction mapping.
\end{abstract}

Keywords: Contraction mapping; image compression; partitioning.

\section{Introduction}

With the expansion of internet activities the digital data usage has risen significantly all over. There seems an umbrella of techniques frequently deployed to store and transmit high resolution images quickly and efficiently [Diane (2014)]. Banach contraction mapping principle is a fundamental theorem in non-linear functional analysis which in the form of Banach fixed point theorem is useful in existence and uniqueness theory. Boxer extended Banach Contraction principle to digital spaces and put forth several notions of usual topology to its digital versions [Boxer (1994), Boxer (1999)]. Various data compression techniques are available in literature [Jayasankar et. al. (2021)]. Contraction mapping principle is useful in digital data compression. A Fractal compression technique has gained lot of attention from research community. It is based on the theory of Iterated Function System (IFS) and was formulated by Hutchinson and Barnsley [Hutchinson (1981), Barnsley (1988a), Barnsley (1988b)] and later on implemented by Jacquin [Jacquin (1992)]. In this the final decompressed image is only an approximation to the original image and thus, fractal compression is often referred as lossy method for image compression. Here image is first partitioned into many mutually exclusive and collectively exhaustive range blocks and domain blocks of larger size and are overlapping. Thereafter, for each range block, suitable contractive mapping and appropriate domain block is determined. Note that such domain block is comparatively larger in size than that of the range block so as to ensure that the mapping is contractive. Later, when the definite contractive mapping is applied to such domain block, it get transformed to the respective range block that might not exactly be same as the original one but is almost similar; some predefined dissimilarity tolerance is subsequently involved for process efficacy evaluation. For elaborate description readers are suggested book by Yuval Fisher [Fisher (1994)].

Creating large number of domain blocks is the key since this enhance chance of finding a near perfect match for given range block. Thus, better understanding towards identification prospects of contraction mapping for range blocks is much necessitated. Therefore, many partitioning techniques are now available and upgraded ones are continuously put forth by the researchers [Diane (2014), Fisher (1994), Bosilj et. al. (2018), Yuan and Cai (2019)]. This work proposes and demonstrates a novel technique contributing in same direction. Presented 
mathematical approach and related analyses intrinsic in determining better suitable contraction mapping by the application of Banach Contraction Principle [Banach (1922)] and its digital versions [Boxer (1994), Boxer (1999), Ege and Karaca (2015)].

\section{Preliminaries}

Definition 2.1: [Banach (1922)] $(X, d)$ be a complete metric space, a mapping T: $X \rightarrow X$ is said to be contraction if $\exists \kappa \in[0,1)$, such that $d(\mathrm{~T} x, \mathrm{~T} y) \leq \kappa d(x, y) \quad \forall x, y \in X$

here $\kappa$ is the contractivity factor. A set of contractive transformations $\mathrm{T}_{i}$ in union with complete metric space $(X, d)$ constitutes an iterated function system (IFS), where the contractivity factor of $\mathrm{T}_{i}$ is $\kappa_{i}, i=1,2 \ldots \ldots N$

Definition 2.2: Let $(X, d)$ be a metric space, if every Cauchy sequence in $X$ is convergent in $X$, then the space $X$ is said to be a complete metric space.

Definition 2.3: The Hausdorff Space of $X$ is defined as $H(X)=\{B \subset X: B$ is compact $\}$

Theorem 2.1: [Ege and Karaca (2015)] Banach Fixed Point Theorem for Digital Contraction Mapping: Let $(X, d, k)$ be a digital metric space and consider a self mapping $f:(X, d, k) \rightarrow(X, d, k)$, if there exists a constant $\lambda \in[0,1) \forall x, y \in X$, we have $d(f(x), f(y)) \leq \lambda d(x, y)$ then the mapping $f$ is called a digital contraction mapping.

Definition 2.4: Peak Signal-to-noise ratio, PSNR, is measured in decibels dB, which calculates the difference between two images and is defined as

$$
P S N R=10 \log _{10}\left(\frac{b \times b}{r m s}\right)
$$

In this paper 8 bit test image is used for illustration, therefore $b=255$.

\section{Partitioning}

Various partitioning methods for digital images are widely available in literature. In this section some crucial partitioning methods for Domain-range blocks analysis (Fig. 1) are mentioned [Jacquin (1992), Fisher (1994), Reusens (1994), Bosilj at. el (2018)]. Quadtree partitioning is based on dividing the image into squares of variable sizes and subsequently finding the best match of each in domain-range block pair with upper limit of tolerance difference. If the best match of some block is not found, then it is further divided into four (quad) smaller blocks of equal size and each smaller block is searched for best match. This process is repeated unless the best match is found with upper tolerance of difference or the partitioning reaches to quad depth. In order to be accurately matched with domain block, typically more detailed areas in the original image require smaller sized range blocks [Fisher (1994)]. Horizontal-Vertical (H-V) Partitioning is comparatively flexible partitioning scheme which uses straight lines to divide the image into rectangles of variable size up to the required depth (not necessarily quad). Process of searching by repeated partitioning ceases once the best domain-range pair is found with given tolerance set [Fisher (1994)]. The triangular partitioning divides the image into two triangles with diagonal, each of which is then subdivided into four triangles by connecting the midpoints of three sides and the process is to be followed recursively until the best match is found [Falconer (1952), Fisher (1994), Yuan and Cai (2019)].
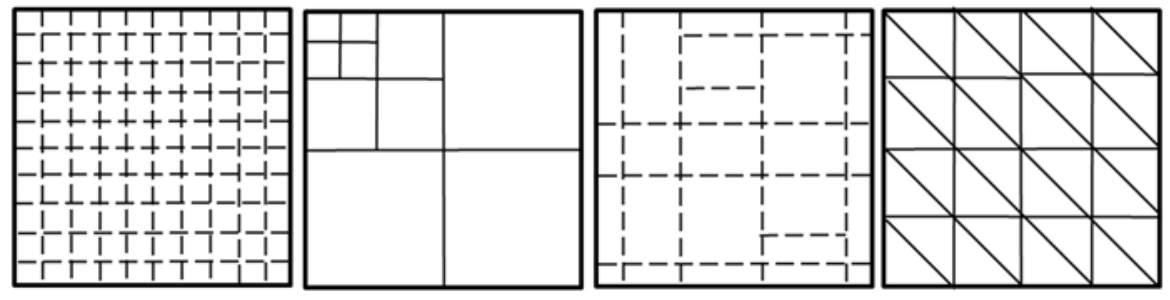

Fig. 1: (a) Fixed width, (b) Quadtree, (c) H-V, (d) Triangular Partitioning

\subsection{Typical partitioning technique}

Consider $x_{1}, x_{2} \in I$ (image space), partitioning curve $C$ is a continuous curve that connects any two points on the (image) domain boundary (usually straight line) and is defined by the method of partitioning adopted. For e.g. partitioning the image along the most significant horizontal or vertical edge is the basis of $\mathrm{H}-\mathrm{V}$ partitioning. A distinctive horizontal partitioning in the image is one which corresponds to a substantial variation in pixel values 
from one row to another. For most distinguishing horizontal line, the maximum difference calculated is considered, however neglecting those which constitute extremely slender rectangles. The process is initiated by calculating the average difference between the rows of pixel between each $i^{\text {th }}$ and $(i+1)^{t h}$ rows as

$$
\frac{\sum_{j} r_{i, j}-\sum_{j} r_{i+1, j}}{M}, 1 \leq i \leq N ; 1 \leq j \leq M
$$

\subsection{Novel approach for partitioning}

This paper proposes a novel understanding of partitioning as a consequence of contraction mapping. For this, we consider a metric $\rho\left(x_{1}, x_{2}\right)$, the partitioning curve $C$ and let the point of intersection of metric with $C$ be denoted by $P\left(\rho\left(x_{1}, x_{2}\right), C\right)$ or simply $P(\rho, C)$ (Fig. 2$)$.

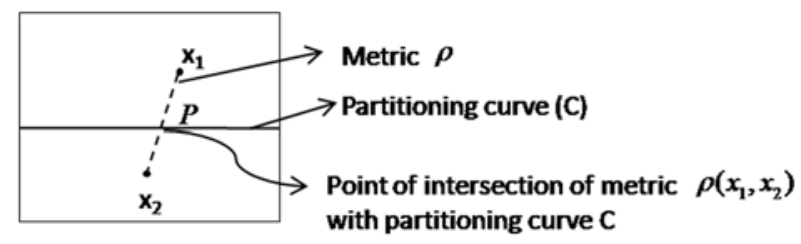

Fig. 2: $P(\rho, C)$

The digital image is an array of pixels; consider the neighboring pixels having almost similar shade of color (associated with individual pixel values) so that they form a cluster (Fig. 3) consisting of finite pixels $x_{i}, i=$ $1,2 \ldots n$. Let the respective floor function of centre of distribution of each cluster denoted by $L_{i}$ the fixed point for that cluster. However, the pixels having drastic shade variation facilitate in deciding the partitioning curve. Now the union of all such clusters (may be overlapping) constitutes the whole image.

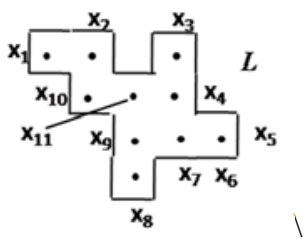

Fig. 3: Cluster of points

Our initial preference is to use simple partitioning with $\mathrm{C}$ as a straight line. Now search for cluster of points (pixels) in image and attempt to draw $C_{s}$ that encompass minimum area with the inclusion of particular cluster. Let $d_{C}$ with subscripts $1,2,3$ etc be the minimum distances of partitioning curve $C$ from the individual points in particular cluster (Fig. 4) with $\mathrm{N}$ number of points. We now propose considering centre of distribution (L) and minimum distance of a point from the $\mathrm{L}$ as $d_{\min }$ in specific manner now described.

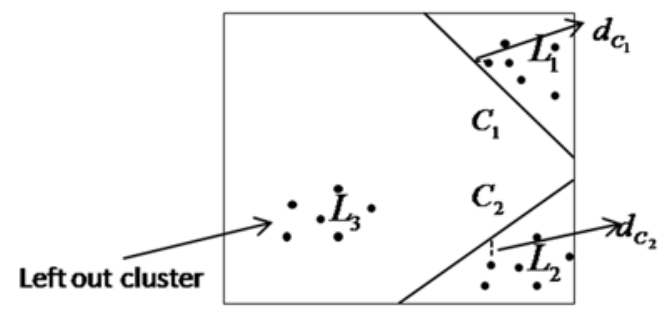

Fig. 4: Distance of $\mathrm{L}$ from cluster

Refer Fig. 2 and assume that points $x_{1}$ and $x_{2}$ corresponds to $L_{1}$ and $L_{2}$. Consequently, $\rho\left(x_{1}, x_{2}\right)$ is now divided into two namely $\rho\left(x_{1}, C\right)$ and $\rho\left(C, x_{2}\right)$. Here, we carefully introduce a factor - metric maneuverability $M_{\rho}$, for $\mathrm{N}$ points in $\mathbb{R}^{2}$ space $M_{\rho}={ }^{\mathrm{N}} \mathrm{C}_{2}$ (C is the combination). Factor $M_{\rho}$ decreases with partitioning and eases the compression process. For higher dimensional space (say $R^{k}$ in general, $M_{\rho}={ }_{k}^{N} C$. This clearly indicates higher/better advantages of partitioning as $\mathrm{k}$ raises its value. For $k=3$ (three dimensional spaces) and $k=4$, four dimensional spaces involving time vector are inclusive in current argument.

Currently, quite reasonably, it can be believed that with an effort of larger scale, proposed concept can also be extended to partitioning curves of different nature (i.e. polynomial curves of degree $\geq 2$ ). A point of caution, however, is that $\mathrm{C}$ represents the polynomial with higher degree (even above one) may have fundamental issue 
that their inverse function leads to multiple solutions during occurrence of transformation. This may require selections or development of (inverse) criterion for taking decision on selecting right solution values to continue with inverse algorithms (for retrieving original image information). It will definitely increase the volume of data to be interpreted. It is not covered in this paper as the formulation is yet to be satisfactorily tested.

Returning to issue of interest, the argument for image partitioning of an image is the certain basic features within image that are radically present in regions of image. These distinct features can be appropriately used for discretizing the image on larger scale thus leading to partitioning.

Thus, it may well assumed certain feature is distinctively present in one partition of image; while another partition might be involving a feature different from the previous one. Such characterizing features may be represented using Lipchitz (functions, properties).

- Let $f_{1}, f_{2}, f_{3}$ etc be Lipschitz such that $f_{1}, f_{2}, f_{3}: X \rightarrow X$, then $f_{1}$ be characterizing partition- 1 of image while $f_{2}$ be characterization partition- 2 of image and so on.

- The proper identification of Lipschitz function in image space can lead to appropriate partitioning strategy for given image.

- Occurrence of more than one function $\left(f_{1}, f_{2}\right.$ etc) in image space signifies some level of discontinuity from the perspective of individual functions i.e. partition space characterized by function $f_{1}$ (say) might have discontinuous $f_{1}$ at partitioning line or curve.

- This simply means characteristic function in one partition space may experience discontinuity in mathematical sense or significant jump in value towards/at partition curve.

- This viewpoint may be extended to various functions $\left(f_{1}, f_{2}\right.$ etc) in different partition space.

- Individual partitions can be treated as closed and bounded from metric space point of view with regard to non-empty set $X$. Let $A \subset \mathbb{R}^{2}$ also belongs to a partition in image space.

- Thus there also occurs $\varepsilon$-neighbourhood of $A$ represented by $S_{A, \varepsilon}$ (Fig. 5). If $A$ is closed and bounded subset of $\mathbb{R}^{2}$ then $S_{A, \varepsilon}$ is also closed and bounded.

- Now Hausdorff space $H(X)$ can be defined considering individual partitions of image.

Let $A, B \in H(X)$, then Hausdorff metric can be appropriately defined as

$$
h(A, B)=\max \left\{\inf \left\{\varepsilon: B \in S_{A, \varepsilon}\right\}, \inf \left\{\varepsilon: A \in S_{B, \varepsilon}\right\}\right\}
$$

where $S_{A, \varepsilon}$ is the $\varepsilon$-neighbourhood of $A$ and $S_{B, \varepsilon}$ is the $\varepsilon$-neighbourhood of $B$

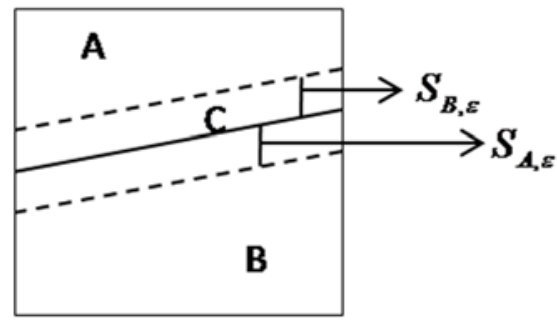

Fig. 5: $\varepsilon$-neighbourhood

- Thus $S_{A, \varepsilon}, S_{B, \varepsilon}$ holds not only the key for identification of partition curve but also for other characterizations of partition space. This provides better flexibility for simultaneous considerations of more than one Lipschitz (i.e. $f_{1}, f_{2}, f_{3}$ etc.) and possibly a way to connect or collectively interpret more partition space. (There seems little reason, at least at this stage, to doubt as why not all partitions in an image handled simultaneously).

Here we appropriately treat image properties like multiple colors, contrasts, brightness etc. Each property is identified through functional information or distribution. Property related information can be adjusted and checked for partitioning possibilities. Related descriptions favor functions that are continuous on $\mathbb{R}^{2}$. Often a region $\mathrm{A}$ of $\mathbb{R}^{2}$ is considered to be closed and bounded subset of $\mathbb{R}^{2}$. We can also consider many subsets of $\mathbb{R}^{2}$ each of which are closed and bounded. Let their union, $\cup_{i=1}^{n} A_{i}$ be completely describing the input image (region). Indicated union operation ascertains flexibility that $A_{i}{ }^{\prime} s$ may be overlapping each other but in the proposed algorithm the overlapping aspect of the clusters is not considered (but left for future work).

\section{Application}

Methodology is illustrated through algorithm given below. 
The following discrete steps are involved:

1. Input a grey image $I$

2. Convert $I$ to pixel values $p_{i, j} \in[0,255]$, zero being black, 255 being white in between there are shades of grey.

3. The pixel value of each cell is compared with at most $n^{2}$ neighboring cells ( $n$ each horizontally and vertically) and if the value matches to tolerance $t$, a cluster 1 is engraved out with partitioning curve $C_{1}$

4. The process is repeated unless the last cell is reached

5. If any cluster $\mathcal{C}_{r} \subset \mathcal{C}_{s}$, then reject $\mathcal{C}_{r}$

6. Find the centre of distribution $(L)$ of all clusters individually.

7. Replace $p_{i, j}$ with $L \forall i, j \in \mathcal{C}_{r}$.

8. With new pixel values construct the image.

9. Output image $I^{\prime}$.

While forming the clusters, the outliners (if any) in the adjacent positions are not included in any of the clusters and will be left unprocessed. The composition of mappings are used and the centre of distribution is defined by the function $f: \mathbb{I} \rightarrow \mathbb{I}, \in[0,255]$, given as $f\left(A_{i}\right)=f\left(g\left(A_{i}\right)\right)=B_{i} \forall A_{i} \in \mathcal{C}$, where $A_{i}=\cup p_{i, j}$ is the set of all pixels in a cluster $\mathcal{C}_{i}, \mathcal{C}=\left\{\mathcal{C}_{1}, \mathcal{C}_{2} \ldots \ldots \mathcal{C}_{q}\right\}, q=$ number of clusters, $\mathcal{f}=$ floor function

$$
g\left(A_{i}\right)=\frac{1}{N} \sum_{1}^{N} x_{i} \forall x_{i} \in A_{i}
$$

$f$ is a contraction on the non-empty set of pixel points $p_{i, j} \in[0,255]$. The distance between two clusters is calculated in Root Mean Square sense, metric $\rho$ is taken to be the quality metric PSNR.

Each pixel in a cluster (region) is similar with respect to some characteristic or computed property, such as color, texture or intensity. Human vision is receptive to edges hence keeping the factor $n$ small will preserve the informative edges. Above algorithm is implemented on various $64 \times 64$ test images (Fig. 6a, b, c). Steps 1, 2, 8 and 9 of the algorithm are implemented using python on spyder IDE, remaining of the computation is done on MS excel. The algorithm can be tweaked or modified to suit typical software like python language and digital images with parametric variety can be processed.

\section{Result and analysis}

Fig. 6a, b, c displays the test image $I$ (original) and 7a, b, c and 8a, b, c are the corresponding output images $I^{\prime}$ (compressed or data reduced). The result obtained for the test images taking $n=8, t=30$ then taking $n=8$, $t=10$ is summarized in the table 1 .

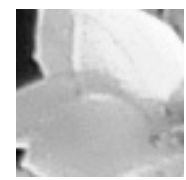

Fig.6 Test image

a

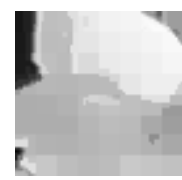

Fig.7 Output image

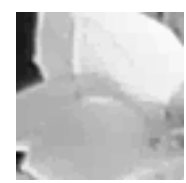

Fig.8 Output image

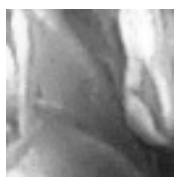

$\mathrm{b}$

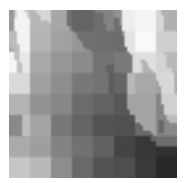

$\mathrm{b}$

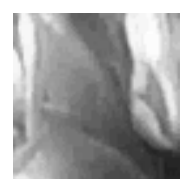

b

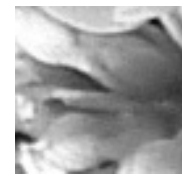

c

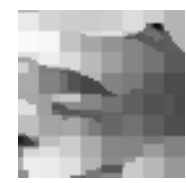

c

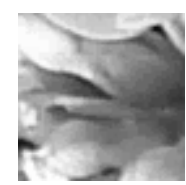

$\mathrm{c}$ 


\begin{tabular}{|c|c|c|c|c|}
\hline & Figure & $\begin{array}{c}\text { Space } \\
(\mathrm{KB})\end{array}$ & $\begin{array}{l}\text { No. of } \\
\text { clusters }\end{array}$ & PSNR \\
\hline \multirow{3}{*}{ Test Image } & $6 a$ & 12.4 & -- & -- \\
\hline & $6 b$ & 12.7 & -- & -- \\
\hline & $6 c$ & 13 & -- & -- \\
\hline \multirow{3}{*}{$\begin{array}{c}\text { Output } \\
\text { Images } \\
n=8, t= \\
30\end{array}$} & $7 a$ & 0.73 & 134 & 33.37 \\
\hline & $7 b$ & 0.54 & 95 & 25.33 \\
\hline & $7 \mathrm{c}$ & 0.68 & 125 & 23.48 \\
\hline \multirow{3}{*}{$\begin{array}{c}\text { Output } \\
\text { Images } \\
n=8, t= \\
10\end{array}$} & $8 \mathrm{a}$ & 1.50 & 343 & 39.71 \\
\hline & $8 b$ & 1.94 & 528 & 39.66 \\
\hline & $8 c$ & 2.22 & 586 & 39.34 \\
\hline
\end{tabular}

Table1: Test summary

The method is lossy which means the output image is approximate to the original image that is the matrix representing $I^{\prime}$ is close to matrix of $I$. Quality of the output images when compared to the corresponding originals is obtained as PSNR. Preliminary inquiry indicates that increasing the number of clusters by decreasing $n$ and/or tapering the tolerance $(t)$ is likely to improve the PSNR but will not be space efficient. For optimal partitioning curve, optimum number of clusters is required both in terms of storage space and time consumption during search process for clustering and image reconstruction.

\section{Conclusion}

An algorithm for partitioning the digital images into clusters of variable sizes that takes into account factors $n$ (dimension of square block of pixels) and $t$ (tolerance in terms of the range of pixel values within the square block) has been developed based on Contraction mapping. The centre of distribution of the cluster is defined such that it facilitates partitioning and assists in compression process. The results indicate implementation success for the proposed algorithm. Comparison of input and the output images demonstrate reduction in image data by preserving the important content and structures of the original image as anticipated. The method is lossy, though. Novel algorithm has potential to downsize the larger sized images to suit data streaming for smaller display gadgets like phones, tablets etc.

\section{References}

[1] Banach, S. (1922): Sur les Op'erations Dans les Ensembles Abstraits et Leur application aux 'equations int'egrales, Fundamenta Mathematicae, (3), pp. 133-181

[2] Barnsley, M. F. (1988): Fractals everywhere, Academic Press, New York.

[3] Barnsley, M. F.; Sian, A. A. (1988): Better way to compress images, BYTE, pp. 215- 223.

[4] Bosilj, P.; Kijak, E.; Lefèvre S.(2018): Partition and Inclusion Hierarchies of Images: A comprehensive survey, J. of Imaging, 4 (2), pp.1-31.

[5] Boxer, L. (1994): Digitally continuous functions, Pattern Recognition Letters, 15, pp. 833-839.

[6] Boxer, L. (1999): A classical construction for the digital fundamental group, J. Math. Imaging Vis., 10, pp. 51-62.

[7] Diane, W. N. N; Xingming, S.; KueMoise, F. (2014): A survey of partition-based techniques for copy-move forgery detection, The Scientific World J., Article ID 975456, 13 pages.

[8] Ege, O.; Karaca, I.(2015): Banach fixed point theorem for digital images, J. Nonlinear. Sci. Appl., 8, pp. $237-245$.

[9] Falconer, K.(1952): Fractal geometry: Mathematical foundations and applications, John Wiley, New York.

[10] Fisher, Y. (1994): Fractal image compression-Theory and applications", Springer Verlag, New York.

[11] Hutchinson, J. E. (1981): Fractals and self-similarity, Indiana Univ. Mathematics J., 35 (5), pp. 713-747.

[12] Jacquin, A. E. (1992): Image coding based on a fractal theory of iterated contractive image transformations, IEEE Transactions on Image Processing, 1 (1), pp. 18-30.

[13] Jayasankar, U.; Thirumal, V.; Ponnurangam D. (2021): A survey on data compression techniques: From the perspective of data quality, coding schemes, data type and applications, J. of King Saud Univ. - Computer and Information Sciences, 33, pp. 119-140.

[14] Reusens, E. (1994): Overlapped Adaptive partitioning for image coding based on the theory of iterated function systems, IEEE proceedings of ICASSP, 5, pp. 569-572.

[15] Yuan, X.; Cai, Z. (2019) "An adaptive triangular partition algorithm for digital images, IEEE Transactions on Multimedia, 21 (6), pp. $1372-1383$ 